\title{
GENETIC ALGORITHM BASED CORPUS OPTIMIZATION TECHNIQUE FOR CONTINUOUS USER AUTHENTICATION
}

\author{
Sonu Agrawal ${ }^{1}$, Sushil Kumar² and Sanjay Kumar ${ }^{3}$
}

\begin{abstract}
Face recognition has been quick growing and challenging field of real time application. Many research works has been done in the area of face recognition with aging and poses. This paper presents a novel approach for optimization of corpus automatically. The proposed work consists of two major stage verification and updation. In verification stage the unknown image with different age of human face is taken and considerable amount of feature have been extracted, these extracted feature are matched with vast corpus of human face images. If the feature matches with the corpus, then to support updation process the extracted features from unknown image are average with the features which is already store in the corpus and store back in the corpus. The updation process has been with the best fit matching using Genetic algorithm. For this an algorithms known as GABAOT (Genetic Algorithms based Automatic Optimization Techniques) has been proposed in the present paper. The algorithm has been tested with the 10 subject of varying age in the present work. Thorough discussion has been done in the present paper along with the proposed algorithms for automatic updation of corpus. The result has been found very satisfactory.
\end{abstract}

Keywords - Artificial Neural Network (ANN), Genetic Algorithms (GA), Optimization Techniques, Ageing, AFR.

\section{INTRODUCTION}

The study of biometry is changing into vital in recent years. Many security applications area unit developed supported biometric personal identification like computerized access management. With personal identification, identity of a private are often determined, preventing unauthorized access of vital knowledge. Most of the biometry system used for this type of applications are face recognition, speech, iris, fingerprint, signatures, and palm print. Among these systems, face recognition would be addressed here as a result of its acceptance and wide usage within the field of security application.

In the large database face recognition provides a convenient way to identify and recognize a person. Using face recognition, we will identify or verify an individual by simply taking a photograph of that person. User now not has to scan his fingerprint or iris for private identification however simply ought to change front of a camera. The system will check its information to identify or verify the person from his image .The present work has been carried out in two phases: Verification and Updation stage. In the verification stage, a knowledge-based model or FACE-MODEL has been framed using ANN by extracting relevant features of known face image. In the same stage the unknown image with different age of human face is taken and considerable amount of feature have been extracted, these extracted feature are matched with vast corpus of human face images. In the understanding phase, this knowledgebased model or corpus has been utilized for the recognition of face recognition using genetic algorithm.

\footnotetext{
${ }^{1}$ Bhilai Institute of Technology, Durg, Chhattisgarh, India, agrawalsonu@ gmail.com

${ }^{2}$ Pragati College of Engineering and Management, Raipur, Chattishgarh, India, sk1_bit@gmail.com

${ }^{3}$ SoS Pt. Ravishankar University, Raipur, Chhattisgarh, India, sanraipur@yahoo.com
} 
Pose and illumination variations will affect the performance of face recognition. Feature Extraction and Dimensionality Reduction was applied using Principal Component Analysis(PCA) and Linear Discriminant Analysis(LDA). During Recognition phase different classifiers such as ANFIS(Adaptive Neuro-Fuzzy Inference Engine), NN(Neural Network), SVM (Support Vector Machine), K-NN(K-Nearest Neighborhood) algorithms were used to the analyze and evaluate the Recognition Rate. R. Raja [19, 20] Most of the work has been carried out using statistical methods and traditional mathematical approaches. Remarkable and good results have been obtained using various components of soft computing and other statistical method. Little amount of work has been carried out when the human-face of the subject is parallel to the image plane, for the recognition of the subject, using hybrid approaches of soft-computing technique. R. [16,17] Hybrid of soft-computing technique means combination of tools of soft-computing. Also the present research work has been carried out by using high-end computing techniques. High-end computing techniques involve combination of soft-computing, symbolic computing. Soft-computing techniques involve artificial neural networks, fuzzy set theory and genetic algorithms. Symbolic computing involves special data type called symbolic objects. Such computing allows researchers to perform mathematical operations without calculating numeric valves. The analytical symbolic computations also involve differentiation, partial differentiation, definite and indefinite integration, taking limits. Integral transformation, Symbolic objects presenting symbolic variables, symbolic numbers, symbolic expressions and symbolic matrices. Earlier very few contributions have been made using neuro-genetic based approach for the recognition of human-face of a subject from side-view (parallel to the image plane). The mechanism that was applied was fully 2-D transformation method with a step of five degree switching reducing step or regressive strategy process.

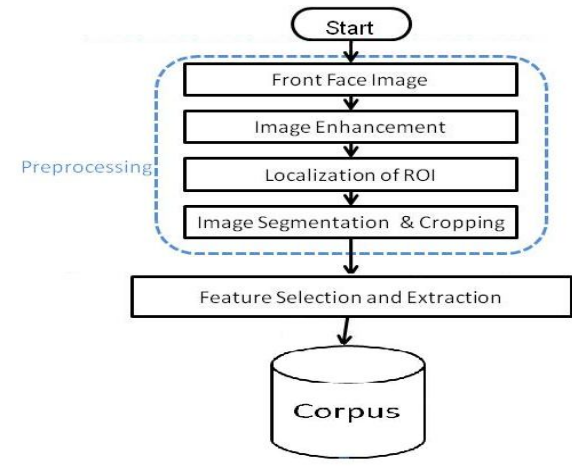

Figure 1: Schematic Diagram for FACE_MODEL

From literature, it has been observed that very little amount of work has been carried out using Genetic approach for the automatic updation of corpus. The schematic diagram for the formation of knowledge-based model, that is, FACE_MODEL has been shown in Figure 1. In Fig.1 it has been shown that a known image has been fed as input. Then it has been pre-processed for enhancement and segmentation. The enhancement has been done for the filtering any noise present in the image. Then it has been segmented using normalized cut method. Discrete Cosine Transform (DCT) has been segmented using employed for loss less compression. Hence the region of Interest has been detected and the relevant features have been extracted. A proper matching-classification-decision process has been carried out using the datasets created in the present research work.

Ming-Hsuan Yang ${ }^{1}$ et.al. done exhaustive study in Numerous techniques that have been developed to detect faces in a single image. The purpose of his paper is to categorize and evaluate these algorithms.

Jae Young $\mathrm{Choi}^{2}$ and others provided an idea to cope with low-resolution faces. They demonstrate that facial color cue can significantly improve recognition performance compared with intensity-based features. They also proposed a new metric called "variation ratio gain" (VRG) to prove theoretically the significance of color effect on lowresolution faces within well-known subspace FR frameworks.

Shengcai Liao[10] et. al proposed a general partial face recognition approach that does not require face alignment by eye coordinates or any other facial points. They developed an alignment-free face representation method based on Multi-Keypoint Descriptors (MKD), where the descriptor size of a face is determined by the actual content of the image. In this way, any probe face image, holistic or partial, can be sparsely represented by a large dictionary of gallery descriptors.

The paper has been organized in the following manner, section II proposes the problem formulation and solution methodology, section III describes the experimental results and discussions, section IV gives the concluding remarks and further work and finally section $\mathrm{V}$ incorporates the references associated with the present work.

\section{PROBLEM FORMULATION AND SOLUTION METHODOLOGY}

In the present paper the problem has been formulated in five stages. The first stage of the problem is the detection of noises and its removal from the image for better performance of the system. The second stage of the problem is detected of boundaries or contours of the image. The third stage of the problem is the selection and extraction of relevant features from the enhanced and segmented image. The fourth stage of the problem is framing of 
knowledge-based model as corpus. The fifth stage of the problem is the recognition problem, given a face image, the goal is to formulate an algorithm that matches the best pattern stored in the Knowledge-based model for classification. The objective of this paper is to investigate and develop a new method for face recognition system using genetic approach. This paper aims to analyze and discuss experimentally the aforesaid problem in subsequent subsections.

\section{A. Mathematical Analysis}

Based on the assumption that the original image is additive with noise. To compute the approximate shape of the wavelet (i.e., Any real valued function of time possessing some structure), in a noisy image and also to estimate its time of occurrence, two methods are available, first one is a simple structural analysis and the second one is the template matching technique. For the detection of wavelets in noisy image, assume a class of wavelets, $\mathrm{S}_{\mathrm{i}}(\mathrm{t}), \mathrm{I}=$ $0, \ldots \mathrm{N}-1$, all having some common structure. Based on this assumption that noise is additive, then the corrupted image has been modeled by the equation -:

$\mathrm{X}(\mathrm{m}, \mathrm{n})=\mathrm{i}(\mathrm{m}, \mathrm{n})+\mathrm{G} \mathrm{d}(\mathrm{m}, \mathrm{n})$

where $i(m, n)$ is the clean image, $d(m, n)$ is the noise and $G$ is the term for signal-to-noise ratio control. To de-noise this image, wavelet transform has been applied. Let the mother wavelet or basic wavelet be $\psi(\mathrm{t})$, which yields to,

$\psi(\mathrm{t})=\exp \left(\mathrm{j} 2 \pi \mathrm{ft}-\mathrm{t}^{2} / 2\right)$

Further as per the definition of Continuous Wavelet transform CWT $(\mathrm{a}, \tau)$, the relation yields to,

$\operatorname{CWT}(\mathrm{a}, \tau)=(1 / \sqrt{a}) \int x(t) \psi\{(\mathrm{t}-\tau) / \mathrm{a}\} \mathrm{dt}$

The parameters obtained in equation (3) has been discretized, using Discrete Parameter Wavelet transform, DPWT (m, n), by substituting a $=a_{0}^{m}, \quad \tau=\mathrm{n} \tau_{0} a_{0}^{m}$. Thus equation (6) in discrete form results to equation (7),

$\operatorname{DPWT}(\mathrm{m}, \mathrm{n})=2^{-\mathrm{m} / 2} \sum_{k} \sum_{l} x(k, l) \psi\left(2^{-\mathrm{m}} \mathrm{k}-\mathrm{n}\right)$

where ' $m$ ' and ' $n$ ' are the integers, $a_{0}$ and $\tau_{0}$ are the sampling intervals for ' $a$ ' and ' $\tau$ ', $x(k, l)$ is the enhanced image. The wavelet coefficient has been computed from equation (4) by substituting $\mathrm{a}_{0}=2$ and $\tau_{0}=1$.

Further the enhanced image has been sampled at regular time interval ' $\mathrm{T}$ ' to produce a sample sequence $\{\mathrm{i}(\mathrm{mT}, \mathrm{nT})\}$, for $\mathrm{m}=0,1,2, \mathrm{M}-1$ and $\mathrm{n}=0,1,2, \ldots \mathrm{N}-1$ of size $\mathrm{M}$ x N image. After employing Discrete Fourier Transformation (DFT) method, it yields to the equation of the form,

$\mathrm{I}(\mathrm{u}, \mathrm{v})=\sum_{m=0}^{M-1} \sum_{n=0}^{N-1} i(m, n) \exp (-j 2 \pi(u m / M+v n / N)$

for $\mathrm{u}=0,1,2, \ldots, \mathrm{M}-1$ and $\mathrm{v}=0,1,2, \ldots \ldots ., \mathrm{N}-1$

In order to compute the magnitude and power spectrum along with phase angle, conversion from time domain to frequency domain has been done. Mathematically, this can be formulated as, Let $\mathrm{R}(\mathrm{u}, \mathrm{v})$ and $\mathrm{A}(\mathrm{u}, \mathrm{v})$ represent the real and imaginary components of $\mathrm{I}(\mathrm{u}, \mathrm{v})$ respectively.

The Fourier or Magnitude spectrum, yields.

$|I(u, v)|=\left[R^{2}(u, v)+A^{2}(u, v)\right]^{1 / 2}$

The phase angle of the transform is defined as,

$\phi(u, v)=\tan ^{-1}\left[\frac{A(u, v)}{R(u, v)}\right]$

The power spectrum is defined as the square of the magnitude spectrum. Thus squaring equation (6), it yields,

$$
P(u, v)=|I(u, v)|^{2}=R^{2}(u, v)+A^{2}(u, v)
$$


Due to squaring, the dynamic range of the values in the spectrum has been found very large. Thus to normalize this, logarithmic transformation has been applied in equation (6). Thus it, yields.

$$
|I(u, v)|_{\text {normalize }}=\log (1+|I(u, v)|)
$$

The expectation value of the enhanced image has been computed and it yields to the relation,

$\mathrm{E}[\mathrm{I}(\mathrm{u}, \mathrm{v})]=\frac{1}{M N} \sum_{u=0}^{M-1} \sum_{v=0}^{N-1} I(u, v)$

where ' $E$ ' denotes expectation. The variance of the enhanced image has been computed by using the relation,

$\operatorname{Var}[\mathrm{I}(\mathrm{u}, \mathrm{v})]=\mathrm{E}\left\{\left[\mathrm{I}(\mathrm{u}, \mathrm{v})-\mathrm{I}^{\prime}(\mathrm{u}, \mathrm{v})\right]^{2}\right\}$

The auto-covariance of an enhanced image has also been computed using the relation,

$\mathrm{C}_{\mathrm{xx}}(\mathrm{u}, \mathrm{v})=\mathrm{E}\left\{\left[\mathrm{I}(\mathrm{u}, \mathrm{v})-\mathrm{I}^{\prime}(\mathrm{u}, \mathrm{v})\right]\left[\mathrm{I}(\mathrm{u}, \mathrm{v})-\mathrm{I}^{\prime}(\mathrm{u}, \mathrm{v})\right]\right\}$

Then the power spectrum density has been computed from equation (12),

$P_{E}(f)=\sum_{m=0}^{M-1} \sum_{n=0}^{N-1} C_{x x}(m, n) W(m, n) \exp (-j 2 \pi f(m+n))$

where $C_{x x}(m, n)$ is the auto-covariance function with ' $m$ ' and ' $n$ ' samples and $W(m, n)$ is the Blackman window function with ' $m$ ' and ' $n$ ' samples.

The data compression has been performed using discrete cosine transform (DCT), given below,

$\operatorname{DCT}_{\mathrm{c}}(\mathrm{u}, \mathrm{v})=\sum_{m=0}^{M-1} \sum_{n=0}^{N-1} I(m, n) \cos \left(\frac{2 \pi T(m+n)}{M N}\right)$

Further for the computation of principal components (i.e., Eigen Values and the corresponding Eigen Vectors), a pattern vector $\overline{p_{n}}$, which can be represented by another vector $\overline{q_{n}}$ of lower dimension, has been formulated using (5) by linear transformation.

Thus $\overline{p_{n}}=[M] \overline{q_{n}}$

where $[M]=[I(m, n)]$ for $\mathrm{m}=0$ to $\mathrm{M}-1$ and $\mathrm{n}=0$ to $\mathrm{N}-1$.

and $\overline{q_{n}}=\min ([\mathrm{M}])$, such that $\overline{q_{n}}>0$

Taking the covariance of equation (15), it yields, the corresponding Eigen vector, given in equation (16),

$\bar{P}=\operatorname{cov}\left(\overline{\left.p_{n}\right)}\right.$

and thus $\bar{P} \cdot M_{i}=\lambda_{i} \quad M_{i}$

where ' $\lambda_{\mathrm{I}}$ ' are the corresponding Eigen values.

Segmentation of an image has been performed using Normalized cut method

To compute the net input to the output units, the delta rule for pattern association has been employed, which yields to the relation,

$\mathrm{y}_{\text {-inj }}=\sum_{i, j=1}^{n} x_{i} w_{i j}$

where ' $\mathrm{y}_{\text {-inj }}$ ' is the output pattern for the input pattern ' $\mathrm{x}_{\mathrm{i}}$ ' and $\mathrm{j}=1$ to $\mathrm{n}$. 
Thus the weight matrix for the hetero associative memory neural network has been calculated from equation (18). For this, the activation of the output units has been made conditional.

$\mathrm{y}_{\mathrm{i}}=\left\{\begin{array}{ccc}+1 & \text { if } & y_{-i n j}>0 \\ 0 & \text { if } & y_{-i n j}=0 \\ -1 & \text { if } & y_{-i n j}<0\end{array}\right.$

The output vector ' $y$ ' gives the pattern associated with the input vector ' $x$ '. The other activation function may also be used in the case where the target response of the net is binary. Thus a suitable activation function has been proposed by,

$\mathrm{f}(\mathrm{x})=\left\{\begin{array}{ccc}1 & \text { if } & x>0 \\ 0 & \text { if } & x<=0\end{array}\right.$

Considering two measures, Accuracy and Precision has been derived to assess the performance of the system, which may be formulated as,

Accuracy $=\frac{\text { Correctly } \operatorname{Re} \text { cognized feature }}{\text { Total number of features }}$

Precision $=\frac{T P R}{T P R+F P R}$

where $\mathrm{TPR}=$ True positive recognition and FPR $=$ False positive recognition .

Starting from the FACE_MODEL, which has various states, each of which corresponds to a segmental feature vector. In one state, the segmental feature vector is characterized by eleven parameters. Considering only three parameters: the eye-to-nose: distance, mean, and the standard deviation, the FACE_MODEL is composed of the following parameters

$\mathrm{FM}_{1}=\left\{D_{s 1}, \mu_{s 1}, \sigma_{s 1}\right\}$

where $\mathrm{FM}_{1}$ means a squint model of the first feature vector, $\mathrm{D}_{\mathrm{s} 1}$ means the distance, $\mu_{\mathrm{s} 1}$ means the mean and $\sigma_{\mathrm{s} 1}$ means the standard deviation based on eye-to-nose distance. Let $\mathrm{w}_{\text {accept }}$ and $\mathrm{w}_{\text {reject }}$ be the two target classes representing acceptance and rejection of face image respectively. The clusters of features have been estimated by taking the probability distribution of these features. This has been achieved by employing Bayes decision theory. Let $\mathrm{P}\left(\mathrm{w}_{\mathrm{i}}\right)$ be the probabilities of the classes, such that, $\mathrm{I}=1,2, \ldots \mathrm{M}$ also let $\mathrm{p}\left(\beta / \mathrm{w}_{\mathrm{i}}\right)$ be the conditional probability density. Assume we have an face image represented by the features, $\beta$. So, the conditional probability $\mathrm{p}\left(\mathrm{w}_{\mathrm{j}} / \beta\right)$, which belongs to $\mathrm{j}^{\text {th }}$ class, is given by Bayes rule as,

$\mathrm{P}\left(\mathrm{w}_{\mathrm{j}} / \beta\right)=\frac{p\left(\beta / \mathrm{w}_{\mathrm{j}}\right) P\left(w_{j}\right)}{p(\beta)}$

So, for the class $\mathrm{j}=1$ to 2 , the probability density function $\mathrm{p}(\beta)$, yields,

$\mathrm{P}(\beta)=\sum_{j=1}^{2} p\left(\beta / w_{j}\right) P\left(w_{j}\right)$

Equation (25) gives a posteriori probability in terms of a priori probability $\mathrm{P}\left(\mathrm{w}_{\mathrm{j}}\right)$. Hence it is quite logical to classify the signal, $\beta$, as follows,

If $\mathrm{P}\left(\mathrm{w}_{\text {accept }} / \beta\right)>\mathrm{P}\left(\mathrm{w}_{\text {reject }} / \beta\right)$, then the decision yields $\beta \in \mathrm{w}_{\text {accept }}$ means 'Acceptance' else the decision yields $\beta \in$ $\mathrm{w}_{\text {reject }}$ means 'Reject'. If $\mathrm{P}\left(\mathrm{w}_{\text {accept }} / \beta\right)=\mathrm{P}\left(\mathrm{w}_{\text {reject }} / \beta\right)$, then it remains undecided or there may be $70 \%$ chance of being right decision making. The solution methodology with proposed algorithms has been discussed in the next section for the complete analysis made till now. 


\section{SOLUTION METHODOLOGY}

Proposed GABAOT (Genetic Algorithms based Automatic Optimization techniques)

\section{Main program \{starts\}}

1. Read the unknown face_image.

2. Set the frame counter, fcount $=1$

3. Set the flag for best fit as fbest $=1$

4. Do

5. Read the face_image[fcount]

6. Enhance the image

7. Apply loss-less Compression

8. Compute the Normalized Cut for segmenting the image

9. Crop the image for locating the Region of Interest

10. Compute the relevant features

11. Compute the genetic parameters using the relation as, $\mathrm{UB}=(((\operatorname{mmax}-\mathrm{mmean}) / 2) * \mathrm{~A})+$ mmean

$$
\mathrm{LB}=(((\text { mmean }-\mathrm{mmin}) / 2) * \mathrm{~A})+\operatorname{mmin}
$$

where ' $A$ ' is the pre-emphasis coefficient, mmax is the maximum value and mmean is the mean value and mmin is the minimum value, UB is the upper bound value and LB is the lower bound value

Store the feature vectors in a look-up table.

12. Perform the best-fit matching with the data set of FACE_MODEL (stored in a master file) using Genetic algorithm

13. Compute maximum matching of parameters

14. If true then set the flag fbest $=0$

15. Employ classification for a two-class problem and then make decision

16. End do

17. Display the result with 'ACCEPT' and 'REJECT'

If ACCEPT

Update the corpus

Else

Main program $\{$ ends $\}$

\section{EXPERIMENTAL RESULTS AND DISCUSSIONS}

The proposed Algorithms called GABAOT (Genetic Algorithms based Automatic Optimization techniques) has been tested for data considering 10 subject. The efficiency of Recognizing the face at different age has been kept at a threshold range of $90 \%$ to $100 \%$ and verified from the trained data stored in the master file. For each face image the geometrical feature measurement have been done with the dataset (FACE_MODEL) and hence employed genetic algorithms for the best fit or match.
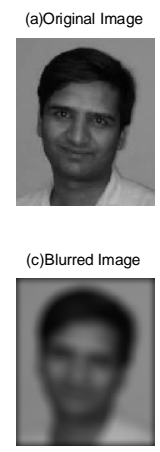

Figure 2. Preprocessing of the captured image.
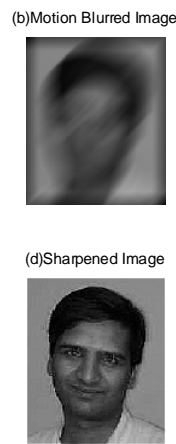

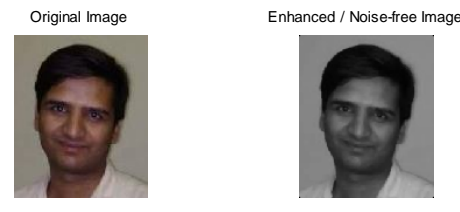

Segmented Image (SI) with Connected Components (CC) Difference of CC and SI
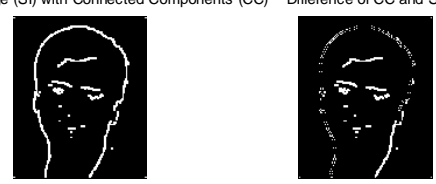

Figure 3. Segmentation of Enhanced noise free image. 


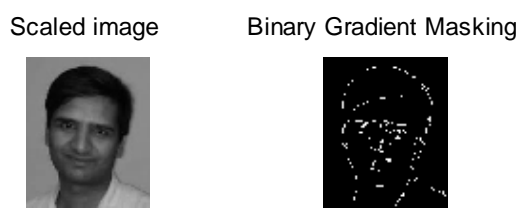

Binary Image with filled holes Cleared Border Image
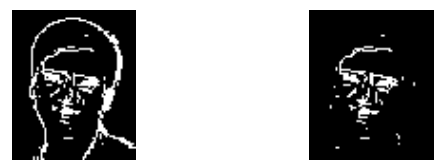

Figure 4. Image scaling and border detection.
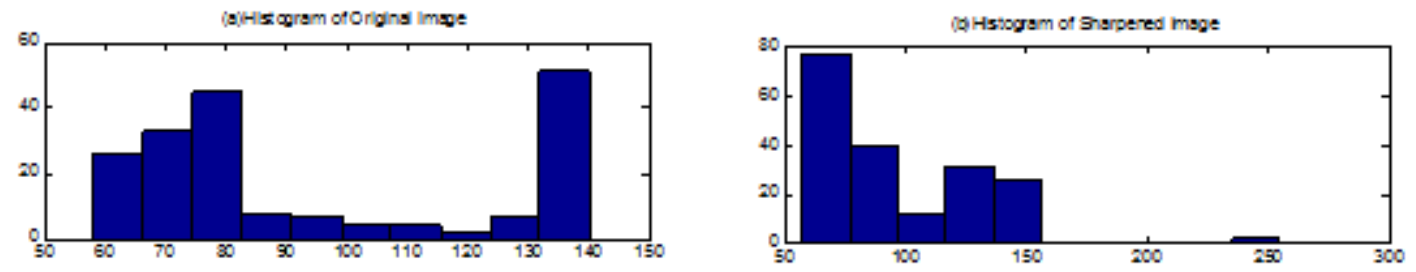

Figure 5. Histogram of orignial and sharpend image.

Table 1 The extracted parameters with respect to facial features of subjects at training phase

\begin{tabular}{|c|c|c|c|c|c|c|c|}
\hline $\begin{array}{c}\text { Image } \\
\text { Files }\end{array}$ & Mean & LB & UB & SD & Auto Corr. & $\begin{array}{c}\text { Eigen } \\
\text { Vector }\end{array}$ & $\begin{array}{c}\text { Eigen } \\
\text { Value }\end{array}$ \\
\hline MO1 & $\mathbf{1 0 . 2 3 4 1}$ & -276.951 & $\mathbf{2 5 8 9 . 4 1}$ & $\mathbf{1 5 7 . 5 1}$ & $\mathbf{2 8 6 6 5}$ & $\mathbf{0 . 0 0 0 4 1 8 9 5}$ & $\mathbf{1 2 9 . 5 0 5}$ \\
\hline MO2 & $\mathbf{9 . 9 2 5 4}$ & $-\mathbf{3 0 1 . 2 5 9}$ & $\mathbf{2 6 2 0 . 9 4}$ & $\mathbf{1 5 2 . 2 5}$ & $\mathbf{4 1 3 6 0}$ & $\mathbf{0 . 0 0 0 1 7 0 8 7}$ & $\mathbf{1 2 8 . 7 4 0}$ \\
\hline MO3 & $\mathbf{1 0 . 1 5 3 4}$ & -242.612 & $\mathbf{2 6 3 7 . 0 8}$ & $\mathbf{1 5 4 . 9 4}$ & $\mathbf{3 0 0 5 0}$ & $\mathbf{0 . 0 0 0 1 8 9 5 6}$ & $\mathbf{7 2 . 3 5 5}$ \\
\hline FO4 & $\mathbf{8 . 3 1 5 6}$ & -290.290 & $\mathbf{2 4 4 8 . 7 3}$ & $\mathbf{1 4 9 . 7 8}$ & $\mathbf{3 6 5 7 5}$ & $\mathbf{0 . 0 0 0 3 5 1 9 8}$ & $\mathbf{6 4 . 9 7 2}$ \\
\hline FO5 & $\mathbf{8 . 6 2 1 3}$ & $-\mathbf{3 1 2 . 6 5 4}$ & $\mathbf{2 5 8 4 . 9 8}$ & $\mathbf{1 5 5 . 1 2}$ & $\mathbf{2 9 3 1 6}$ & $\mathbf{0 . 0 0 0 2 4 1 5 8}$ & $\mathbf{6 2 . 4 7 6}$ \\
\hline
\end{tabular}

Table 2 The extracted parameters with respect to facial features of unknown subjects at verification phase

\begin{tabular}{|c|c|c|c|c|c|c|c|}
\hline $\begin{array}{c}\text { Image } \\
\text { Files }\end{array}$ & Mean & LB & UB & SD & Auto Corr. & $\begin{array}{c}\text { Eigen } \\
\text { Vector }\end{array}$ & $\begin{array}{c}\text { Eigen } \\
\text { Value }\end{array}$ \\
\hline $\begin{array}{c}\text { Unknown } \\
\text { Image }\end{array}$ & 10.2012 & -299.250 & 2619.41 & 150.42 & 41271 & $\mathbf{0 . 0 0 0 1 7 2 8 7}$ & 129.745 \\
\hline
\end{tabular}

As these values were found to be matched with image file MO2 within given threshold value, the unknown image is identified as MO2. However for future identification process these values will be averaged to get better result.

Table 5.3 The extracted parameters with respect to facial features of after updation.

\begin{tabular}{|c|c|c|c|c|c|c|c|}
\hline $\begin{array}{c}\text { Image } \\
\text { Files }\end{array}$ & Mean & LB & UB & SD & Auto Corr. & $\begin{array}{c}\text { Eigen } \\
\text { Vector }\end{array}$ & $\begin{array}{c}\text { Eigen } \\
\text { Value }\end{array}$ \\
\hline MO1 & $\mathbf{1 0 . 2 3 4 1}$ & -276.951 & 2589.41 & $\mathbf{1 5 7 . 5 1}$ & $\mathbf{2 8 6 6 5}$ & $\mathbf{0 . 0 0 0 4 1 8 9 5}$ & $\mathbf{1 2 9 . 5 0 5}$ \\
\hline MO2 & $\mathbf{1 0 . 0 6 3 3}$ & $-\mathbf{3 0 0 . 2 5 5}$ & $\mathbf{2 6 2 0 . 1 7 5}$ & $\mathbf{1 5 1 . 3 3 5}$ & $\mathbf{4 1 3 1 5 . 5}$ & $\mathbf{0 . 0 0 0 1 7 2}$ & $\mathbf{1 2 9 . 2 4 3}$ \\
\hline MO3 & $\mathbf{1 0 . 1 5 3 4}$ & $-\mathbf{2 4 2 . 6 1 2}$ & $\mathbf{2 6 3 7 . 0 8}$ & $\mathbf{1 5 4 . 9 4}$ & $\mathbf{3 0 0 5 0}$ & $\mathbf{0 . 0 0 0 1 8 9 5 6}$ & $\mathbf{7 2 . 3 5 5}$ \\
\hline FO4 & $\mathbf{8 . 3 1 5 6}$ & $-\mathbf{2 9 0 . 2 9 0}$ & $\mathbf{2 4 4 8 . 7 3}$ & $\mathbf{1 4 9 . 7 8}$ & $\mathbf{3 6 5 7 5}$ & $\mathbf{0 . 0 0 0 3 5 1 9 8}$ & $\mathbf{6 4 . 9 7 2}$ \\
\hline FO5 & $\mathbf{8 . 6 2 1 3}$ & $-\mathbf{3 1 2 . 6 5 4}$ & $\mathbf{2 5 8 4 . 9 8}$ & $\mathbf{1 5 5 . 1 2}$ & $\mathbf{2 9 3 1 6}$ & $\mathbf{0 . 0 0 0 2 4 1 5 8}$ & $\mathbf{6 2 . 4 7 6}$ \\
\hline
\end{tabular}

\section{CONCLUDING REMARKS AND FURTHER WORK}

In order to recognize a face whose face differ due to current age and age at the time of registration, a through mathematical analysis have been carried out in present paper. For the current work only front face image has been consider. More over only limited number of facial features has been extracted. Few more features can be extracted 
for achieving better results. In the work explained here only consider few features only. More features can be extracted to achieve better acceptance ratio. Further work can be in the direction of designing a framework so as to identify a person efficiently irrespective of his age.

\section{REFERENCES}

[1] Ming-hsuan yang, david j. Kriegman, narendra ahuja, ieee "detecting faces in images: a survey" ieee transactions on pattern analysis and machine intelligence, vol. 24, no. 1, january 2002

[2] Jae young choi, yong man ro, konstantinos n. (kostas) plataniotis, "color face recognition for degraded face images" ieee transactions on systems, man, and cybernetics_part b: cybernetics, vol. 39, no. 5, october 2009

[3] Zhifeng li, unsang park, anil k. Jain "a discriminative model for age invariant face recognition" ieee transactions on information forensics and security, vol. 6, no. 3, september 2011

[4] Maria de marsico, member, ieee, michele nappi, and daniel riccio "faro: face recognition against occlusions and expression variations" ieee transactions on systems, man, and cybernetics—part a: systems and humans, vol. 40, no. 1, january 2010

[5] Qian tao and raymond veldhuis "biometric authentication system on mobile personal devices" ieee transactions on information forensics and security, vol. 6, no. 3, september 2011

[6] Unsang park, member, yiying tong, member, anil k. Jain "age-invariant face recognition" ieee transactions on pattern analysis and machine intelligence, vol. 32, no. 5, may 2010

[7] Wilman w. W. Zou, pong c. Yuen "very low resolution face recognition problem" ieee transactions on image processing, vol. 21, no. 1, january 2012

[8] Ajay kumar and david zhang "improving biometric authentication performance from the user quality" ieee transactions on instrumentation and measurement, vol. 59, no. 3, march 2010

[9] N. Sudha, a. R. Mohan, pramod k. Meher "a self-configurable systolic architecture for face recognition system based on principal component neural network" ieee transactions on circuits and systems for video technology, vol. 21, no. 8, august 2011

[10] Shengcai liao, anil k. Jain, stan z. Li "partial face recognition: alignment-free approach" ieee transactions on pattern analysis and machine intelligence vol. 2, no. 5, august 2012

[11] Sonu Agrawal, Sushil Kumar, Sangeeta Agrawal, "A Survey on Face Recognition Techniques", in the proceedings of International Journal of Engineering Research and Applications (IJERA - 2012), Volume 2, Issue 4, July 2012.

[12] Sangeeta Agrawal, Rohit Raja, Sonu Agrawal, "Support Vector Machine for age classification", in the proceedings of International Journal of Emerging Trends and Advanced Engineering, Volume 2, Issue 5, May 2012.

[13] Sonam Saluja, Aradhana Kumari Singh, Sonu Agrawal, "A Study of Edge-Detection Methods" International journal of advanced research in Computer and Communication Engineering, Volume 2, Issue 1, January 2013

[14] Pooja Kamavisdar, Sonam Saluja, Sonu Agrawal "A Survey on Image Classification Approaches and Techniques" International journal of advanced research in Computer and Communication Engineering, Volume 2, Issue 1, January 2013

[15] Sonu Agrawal, Sushil Kumar, Sanjay Kumar “A Novel Idea for Age Invariant Face Recognition” International Journal Of Innovative Research In Science, Engineering And Technology (IJIRSET), Volume 3, Issue 8, August 2014

[16] Rohit Raja, Tilendra Shishir Sinha, Ravi Prakash Dubey (2016), "Soft Computing and LGXP Techniques for Ear Authentication using Progressive Switching Pattern", Published in International Journal of Engineering and Future Technology, Vol. 2, Iss. 2, 2016.

[17] Rohit Raja, Tilendra Shishir Sinha, Ravi Prakash Dubey (2016), “Orientation Calculation of human Face Using Symbolic techniques and ANFIS", Published in International Journal of Engineering and Future Technology, Vol. 7, Iss.7, pp. 37-50, 2016.

[18] Rohit Raja, Tilendra Shishir Sinha, Ravi Prakash Dubey (2015), "Recognition of human-face from side-view using progressive switching pattern and soft-computing technique", Association for the Advancement of Modelling and Simulation Techniques in Enterprises, Advance B, Vol. 58, N 1, pp. 14-34, 2015

[19] Tilendra Shishir Sinha, Raj kumar Patra, and Rohit Raja, "A Comprehensive analysis of human gait for abnormal foot recognition using Neuro-Genetic approach”, International Journal of Tomography and Statistics (IJTS), vol. 16, no. W11, pp. 56-73, Winter 2011.

Tilendra Shishir Sinha, Devanshu Chakraverty, Rajkumar Patra and Rohit Raja, “ Modelling and Simulation for the Recognition of Physiological and Behavioural Traits Through Human Gait and Face Images", Discrte Wavelet TransformsA Compendium of New Approaches and Resents Application, by intech China ISBN 978-953-51-0940-2, pp. 95-125, Feb 2013. 